\title{
UN ESTUDIO TRANSVERSAL SOBRE LA PERCEPCIÓN DE LA VITALIDAD ETNOLINGÜÍSTICA EN JÓVENES Y ADULTOS DE CATALUÑA
}

\author{
A CROSS-SECTIONAL STUDY ON ETHNOLINGUISTIC VITALITY \\ PERCEPTION IN YOUNG AND ADULTHOOD PEOPLE FROM CATALONIA
}

\author{
MARIA Àngels VILAdot \\ Universitat Oberta de Catalunya. España \\ maviladot@uoc.edu \\ MoIsĖs EsteBAN \\ Universitat de Girona. España \\ moises.esteban@udg.edu
}

\section{RESUMEN}

El estudio utiliza un diseño transversal en el campo de la percepción de la vitalidad etnolingüística. Es el primero que compara la percepción de la vitalidad etnolingüística, así como los factores asociados, entre adultos jóvenes y adultos, en relación con los grupos castellanohablantes y catalanohablantes de la Comunidad Autónoma de Cataluña. Para ello, se aplicó el "Cuestionario de vitalidad etnolingüística subjetiva" (CVS) a una muestra de 527 participantes, 268 jóvenes y 259 adultos, de los cuales se seleccionó una submuestra de individuos que tenían el catalán como lengua materna y se identificaban como catalanes ( $n=301)$. En ambas muestras se aduce una tendencia a discriminar favorablemente la vitalidad percibida en el grupo catalán, aspecto que se acentúa significativa en el grupo de jóvenes estudiado en relación al grupo de edad de los adultos. Se discuten los resultados según las repercusiones teóricas y pragmáticas de los estudios realizados en el ámbito de la comunicación intergrupal.

\section{Palabras Clave}

Comunicación intergrupal, Diseño transversal, Identidad etnolingüística, Vitalidad etnolingüística percibida.

\section{ABstRact}

The work hereby uses a cross-sectional design in the study field of ethnolinguistic vitality perception. It is the first one to compare ethnolinguistic vitality perception, and its related factors, between young people and adults in relation to the Catalan-speaking and the Spanish-speaking groups in Catalonia. To do so, the subjective ethnolinguistic vitality questionnaire was used on a sample of 527 participants, 268 young and 259 adults, among which there was a sub-sample of individuals who had Catalan as their mother-tongue and identified themselves as Catalan people $(n=301)$. In both the total sample and the sub-sample a tendency to positively discriminate perceived vitality of the Catalan group is alleged, an aspect that is emphasized significantly in the group of emerging adulthoods. Results are discussed based on the theoretical and pragmatic repercussions of studies done on intergroup communication field. 


\section{KEYWORDS}

Cross-Sectional Design, Ethnolinguistic Vitality, Intergroup Communication, Perceived Ethnolinguistic Vitality.

\section{INTRODUCCIÓN}

\section{Vitalidad etnolingüística objetiva (VEL)}

La vitalidad de las comunidades lingüísticas puede afectar la cualidad de la comunicación intergrupal entre hablantes de diferentes grupos de lengua. Este es el caso en el que el acento, el dialecto y la lengua, no solamente proporcionan importantes indicaciones para la categorización de los hablantes, sino que además son dimensiones sobresalientes de la identidad étnica. La vitalidad etnolingüística es definida como "lo que hace posible a un grupo comportarse como una entidad distintiva y colectiva en los contactos intergrupales" (Giles, Bourhis y Taylor 1977:308). Cuanta más vitalidad tiene un grupo etnolingüístico más capacidad tendrá para usar su propia lengua y, por lo tanto, para sobrevivir y prosperar como una entidad colectiva.

Tres variables socio-estructurales influencian la vitalidad de los grupos etnolingüísticos: demografía, apoyo y control institucional, y estatus.

Las variables demográficas hacen referencia al número absoluto de hablantes y su distribución a través del territorio nacional, regional o urbano; por ejemplo, índice de nacimientos y mortalidad, matrimonios mixtos, y los modelos de emigración/inmigración en un territorio ancestral. Los factores demográficos constituyen un activo fundamental de los grupos etnolingüísticos como "la fuerza numérica" y pueden ser utilizados como una herramienta de legitimación para conceder a las comunidades lingüísticas el apoyo y control institucional que necesitan para alentar su desarrollo en el marco multilingüe.

El grado con el que una comunidad lingüística ha conseguido representación formal e informal en las instituciones de una región, estado o nación constituye su apoyo y control institucional. El control institucional se define como el grado de control que un grupo tiene sobre su propio destino. Es decir, el grado de poder social y político que tiene un grupo lingüístico en relación con las comunidades lingüísticas con las que compite (Sachdev y Bourhis 2005). El apoyo institucional se refiere al grado de representación formal e informal que una comunidad lingüística ha conseguido en las instituciones de una región, estado o nación. El apoyo informal se refiere al grado con el que los miembros de una comunidad lingüística están organizados como grupo de presión para representar y salvaguardar los intereses de su propia lengua en los dominios públicos y privados (Sachdev y Bourhis 1993). El apoyo formal se refiere al grado con el que los miembros de un grupo lingüístico han conseguido posiciones de control y decisión en las instituciones privadas y en el gobierno local y nacional. Este control mejora la probabilidad de que la primera lengua será enseñada y usada en la educación, usada como una lengua de cuidados y salud en los hospitales, usada como una lengua de trabajo en los negocios y los servicios públicos, e incluida como la lengua de los medios de comunica- 
ción. En los grupos minoritarios bien organizados políticamente, las lenguas minoritarias a menudo son un vehículo de expresión y, mediante esta organización, pueden ejercer presión en el grupo exterior para proteger sus intereses en situaciones de comunicación transculturales. En un nivel más formal, los grupos que tienen poca representación en el estado, en los negocios, y en la cultura, tienen menos posibilidades de sobrevivir como entidades políticas. Los medios de comunicación como forma de control institucional pueden afectar considerablemente los procesos de cambio de lengua de una comunidad. En nuestro país los veinticinco años de televisión catalana (TV3) son un ejemplo en este sentido. No obstante, las nuevas tecnologías, la Televisión Digital Terrestre (TDT) 0 la televisión por internet aportaran nuevos problemas y nuevas oportunidades para que el apoyo y control institucional pueda canalizar sus esfuerzos en el mantenimiento de la lengua. La educación es un pilar fundamental para el mantenimiento de la lengua. Si en las escuelas se fomenta el aprendizaje de los niños en la lengua minoritaria y se les enseña a leer y escribir en esta lengua sentaremos las bases para contribuir al mantenimiento de la lengua propia en las esferas de uso privado y social (Clément y Bourhis 1996).

El hecho de que la Administración utilice la lengua materna o territorial es un aspecto importante en el mantenimiento del idioma propio de la comunidad. Por ejemplo, la inmigración ha de tener relación con la Administración en el proceso de integración a la nueva comunidad lingüística, y el hecho de que esta comunicación se pueda realizar en la lengua vernácula del territorio de integración y que la información que se les suministre en forma variada de folletos o formularios sea en dicha lengua, motivará a los recién llegados para la adquisición de dicha lengua, puesto que lo percibirán como un modo de integración. Otro ejemplo de apoyo institucional es el programa de parejas lingüísticas (entre un catalán y un recién llegado) que se está desarrollando en Cataluña; dicho programa está demostrando su gran eficacia para el aprendizaje del catalán y la integración armoniosa de los recién llegados. También, que la lengua minoritaria sea la lengua de la religión del país, es un factor para su mantenimiento.

La presencia y la cualidad de los líderes en las instituciones formales e informales representando a los grupos lingüísticos también contribuye al apoyo y control institucional de las comunidades lingüisticas. La ganancia en apoyo y control institucional muchas veces depende de la emergencia de activistas y líderes carismáticos que tienen éxito movilizando a los grupos para luchar para un mayor control institucional y un mayor soporte para el uso de la lengua de su grupo (Harwood, Giles y Bourhis 1994). Los grupos lingüísticos que han tenido ganancias en los factores de apoyo y control institucional también probablemente se beneficiarán de un considerable estatus relativo ante los grupos menos dominantes. Todo lo que se acaba de manifestar pone en evidencia que el apoyo y control institucional es la dimensión por excelencia de la vitalidad etnolingüistica que los individuos de los grupos lingüísticos dominantes utilizan para mantener y afirmar su posición privilegiada en las relaciones con los individuos de otros grupos lingüísticos.

Las variables de estatus son las variables relacionadas con el prestigio social de las lenguas, su posición socio-histórica dentro del estado, y el prestigio local, nacional e internacional de su lengua y cultura. La psicología social muestra de forma evidente que 
los hablantes de grupos de alto estatus disfrutan de una identidad social más positiva y probablemente usarán su lengua en un amplio abanico de situaciones públicas y privadas. Contrariamente, ser miembro de un denigrado grupo lingüístico con bajo estatus puede socavar la esperanza colectiva de la minoría lingüística de mantenerse como una comunidad lingüistica diferenciada, empujándole hacia la asimilación lingüística. La experiencia de pertenecer a una comunidad con alto versus bajo estatus lingüístico es reforzada a través de estereotipos y salvaguardada mediante la adopción de leyes que regulan el estatus relativo de las comunidades lingüísticas en estados multilingües (Bourhis y Maass 2005).

Las tres dimensiones anteriores se combinan para afectar en una dirección u otra la fuerza global de la vitalidad de los grupos etnolingüísticos. Una comunidad lingüística puede ser débil en las variables demográficas, pero fuerte en soporte institucional y en el factor estatus; resultando una posición media de vitalidad, relativamente, a una minoría lingüística débil en las tres dimensiones de la vitalidad (Ros, Huici y Cano 1984).

\section{Vitalidad etnolingüística subjetiva (VELS)}

El verdadero reto del concepto de vitalidad etnolingüística ha sido, sin embargo, proporcionar una valoración "subjetiva" sobre cómo los miembros de las colectividades étnicas juzgan las condiciones sociales que afectan a su grupo y a otros grupos relevantes. De esta forma, la vitalidad percibida puede ser un elemento de lo que Triandis (1994) ha llamado "cultura subjetiva". De hecho, lo que ha hecho avanzar el consiguiente enfoque (complementario) de la vitalidad ha sido el descubrir cómo los índices objetivos de vitalidad están relacionados con sus homólogos subjetivos -mediante el papel mediador de la red lingüística del propio grupo, anunciado por Allard y Landry (1994)-. Para valorar los elementos subjetivos, Bourhis, Giles, y Rosenthal (1981) crearon un instrumento de 22 ítem (el CVS) para cuantificar las percepciones individuales sobre la vitalidad del propio grupo y el exogrupo. Aunque el concepto de vitalidad ha sido a veces controvertido y ha estado sujeto a saludables escrutinios metodológicos y conceptuales, se ha demostrado la gran fiabilidad interna del instrumento para cuantificar la vitalidad subjetiva en su versión inglesa (Abrams, Barker y Giles 2009; Allard y Landry 1994; McCann 2000; Giles, Rosenthal y Young 1985), así como en su traducción y adaptación al contexto catalán (Viladot 1993) permitiendo el estudio sistemático de aspectos como la geolinguística (McConnell 1991), el aprendizaje de segundas lenguas (Baldaquí 2004) y la planificación lingüística (Landry y Allard 1994), con un enfoque especial sobre la forma en que las percepciones de la vitalidad se relacionan con el mantenimiento de la lengua y la solidaridad del grupo etnolingüístico (Giles y Viladot 1994; Viladot 1993; Viladot y Siguan 1992), o con el efecto de un determinado cambio histórico (Atkinson 2000; Cenoz y Valencia 1993; Giles y Viladot 1994; Kraemer 1992; Kraemer, Olshtain y Badier 1994; Komondouros y McEntee-Atalianis 2007). 


\section{Perfiles en la percepción de vitalidad}

Numerosos estudios utilizando el CVS nos muestran que, en determinados contextos, los miembros de los grupos etnolingüísticos tienen una percepción bastante realista sobre la situación de su vitalidad etnolingüística (Harwood, Giles y Bourhis 1994). Esto se ha observado, por ejemplo, entre la segunda generación de inmigrantes italianos en la ciudad canadiense de Hamilton (Bourhis y Maass 2005), entre los jóvenes bilingües en el País de Gales (Giles y Johnson 1987), entre la primera generación de inmigrantes chinos en Toronto (Sachdev y Bourhis 1993) y en Cataluña en jóvenes bilingües que tenían como primera lengua el catalán y se sentían catalanes en primer lugar antes que españoles (Viladot 1993; Giles y Viladot 1994; Viladot et al. 2007; Ytsma, Viladot y Giles 1994).

Sin embargo, otras muchas investigaciones han subrayado que las supuestamente iguales condiciones sociales pueden ser vistas de modos muy distintos por las diversas partes implicadas (Harwood, Giles, y Bourhis 1994). Es decir, estos estudios han mostrado sesgos en las valoraciones que los grupos lingüísticos realizan de la vitalidad propia y de los otros grupos. Por ejemplo, estudiantes anglo-australianos de Melbourne acentuaron las diferencias de vitalidad entre su propio grupo y los greco-australianos, quienes; a su vez, las atenuaron (Giles, Rosenthal y Young 1985). Similares descubrimientos se muestran en el estudio israelí de Kraemer, Olshtain y Badier (1994) con una mayoría de estudiantes judíos y una minoría de estudiantes árabes, así como en el estudio de Karahan (2004) sobre los bosnios-turcos que viven en Sakarya, y el estudio de Komondouros y McEntee-Atalianis (2007) sobre la comunidad greco-ortodoxa de Istanbul. En Hong Kong, apareció un perfil de vitalidad intergrupal más diferenciado, en el que los estudiantes occidentales y cantoneses favorecieron su propio grupo en ítems relacionados con el estatus de la lengua, pero consideraron que el otro grupo tenía significativamente más control y más poder en otras escalas (Young, Giles y Pierson 1986). Además, la comparación de los datos obtenidos en Hong Kong antes de la firma del Tratado chino-británico (1983), con los inmediatamente posteriores, mostró que los chinos percibieron que su vitalidad había incrementado mientras que la vitalidad occidental había disminuido (Young, Giles y Pierson 1986). Sin embargo, se puede argumentar que no hubo cambios significativos (si los hubo) en las vitalidades objetivas de los dos grupos durante este periodo de 18 meses. En Cataluña, jóvenes del grupo "catalán" (identidad catalana y catalán como primera lengua) mostraron una percepción ajustada a la vitalidad objetiva y jóvenes de los grupos "mixto" (identidad catalana y español como primera lengua) y "español" (identidad española y español como primera lengua) mostraron en general una sobreestimación de la vitalidad de los catalano-hablantes (Viladot 1993). También en el contexto español se han distinguido tres prototipos identitarios según la combinación de identidad, lengua y cultura: la Identidad Autonómica, la Identidad Española y la Identidad Autonómica-Española (bilingüe-bicultural); siendo la satisfacción con la identidad cultural y lingüística, el uso de las lenguas y las creencias egocéntricas los antecedentes más potentes de dichas identidades (Azurmendi y Bourhis 1998; Ros et al.1999). Precisamente, las creencias exocéntricas (otros grupos) 
y egocéntricas (el propio grupo) constituyen elementos claves en la VELS (Romay, García-Mira y Azurmendi 1999).

Harwood, Giles y Bourhis (1994:172-175), revisan éstas y otras investigaciones y establecen tres perfiles generales en la percepción de la vitalidad etnolingüística:

Percepción distorsionada a favor de la vitalidad del propio grupo (perceptual distorsions in favor of ingroup vitality). Se caracteriza por el sesgo a favor de la vitalidad del propio grupo tanto en el grupo dominante como en el grupo subordinado, y es debido al hecho de que el grupo dominante acentúa algunas de las diferencias de vitalidad con el otro grupo, mientras que el grupo subordinado las atenúa. Por ejemplo, el estudio citado anteriormente sobre la segunda generación de inmigrantes italianos al Canadá en contextos en que el grupo dominante no es mayoritario.

Percepción distorsionada a favor de la vitalidad del otro grupo (perceptual distorsions in favor of outgroup vitality). Se caracteriza por el hecho que el grupo dominante mitiga las diferencias en vitalidad en relación al grupo subordinado, mientras que el grupo subordinado acentúa las diferencias. El estudio de Sachdev y Bourhis (1993) sobre la segunda generación de chinos en Toronto es un ejemplo de este perfil general en la percepción de la vitalidad etnolingüística.

Falta de acuerdo entre las percepciones de la vitalidad (non consensual vitality perceptions). La diferencia entre las percepciones de la vitalidad etnolingüística de los grupos es muy elevada, tanto en dirección como en grado. En algunos ítems los dos grupos exageran la vitalidad propia (subperfil 1), mientras que en otros ítems exageran la vitalidad del otro grupo (subperfil 2). Un ejemplo es el estudio en Hong Kong (Pierson 1987).

Así pues, la vitalidad no es una construcción social estática sino, más bien, una construcción social maleable que depende del número de miembros de un grupo social y de las circunstancias sociales y políticas cambiantes. En este sentido, Viladot et al. (2007) han demostrado cambios en la vitalidad percibida entre los datos recogidos en 1987 (Viladot 1993) y en 2007. El estudio presenta de forma clara cómo, aun siendo favorable, la percepción de la vitalidad del catalán ha decrecido en el factor estatus y en el factor demolingüístico. Pero, en cambio, en su conjunto, la vitalidad percibida del factor apoyo institucional ha aumentado. Parece que los estudiantes catalanohablantes de 2007 se muestran preocupados por el futuro del idioma y del propio grupo, aunque, en general, se sienten seguros del poder del grupo. Un dato interesante es que estos estudiantes de identidad catalana consideraron que, en el presente, los catalanes, en comparación a los españoles, tienen más fuerza y actividad en Cataluña; en cambio estimaron que serán los castellanohablantes los que tendrán más fuerza y actividad dentro de 20030 años.

Todos estos trabajos se centran en el estudio de las vitalidades subjetivas dentro de un mismo grupo o entre grupos etnolingüísticos. Sin embargo, hasta la fecha no se han realizado estudios con diseños transversales, es decir, análisis de la vitalidad etnolingüística subjetiva en diferentes grupos de edad observados en un mismo momento temporal. Ello permitiría indagar en las diferencias de edad (entre jóvenes y adultos, por ejemplo) en la percepción de la vitalidad etnolingüística de determinados grupos sociales. Es decir, permitiría incluir la variable edad, aspecto que ha recibido poca atención en los estudios sobre vitalidad etnolingüística. 


\section{La posible influencia del factor edad en la vitalidad etnolingüística subjetiva}

Recientemente, algunos teóricos del desarrollo humano han propuesto la diferenciación de dos períodos evolutivos entre los 10 y los 30 años de edad. Se trata de la adolescencia (que iría de los 10 a los 18 años), y la "edad adulta emergente" -nosotros utilizaremos el concepto de juventud- que comprende las personas entre los 18 a los 30 años de edad (Arnett 2000). Según el autor anteriormente mencionado, la juventud es el período de transición en que se pasa de la adolescencia al inicio de la edad adulta en sociedades desarrolladas, como los Estados Unidos o Europa; sociedades, por otra parte, en las que han aumentado el número de divorcios, la incertidumbre en el terreno profesional o en las que se ha retrasado la emancipación familiar, la independencia económica y la edad de casarse y tener hijos (Williams, Garret y Tennant 2004). Existen cinco características que distinguen la juventud de otras edades según Arnett (2000;2007). Estas son: fase de exploración identitaria (las personas exploran varias posibilidades de amor, trabajo, relaciones interpersonales); inestabilidad (en el trabajo, en la vivienda, en las relaciones amorosas); centrarse en uno mismo (empiezan a construir sus propias opiniones independientes de los puntos de vista mantenidos por sus familias); sentirse en medio (ya no se es adolescente pero tampoco plenamente adulto) y, finamente, la edad de las posibilidades (son posibles muchos futuros distintos).

El estudio de Harwood et al. (1994), realizado en California y Hong Kong con tres cohortes de edad: jóvenes, mediana edad y ancianos, pone de manifiesto que la percepción de la fuerza y vitalidad social que tienen los jóvenes en la actualidad es muy baja (comparativamente a la vitalidad que tienen los de mediana edad). En un estudio no publicado, Viladot (1994) observa el mismo patrón perceptivo en la población catalana. Es decir, los jóvenes de la muestra estudiada son percibidos por ellos mismos, por los de mediana edad y por los más mayores con menor fuerza y vitalidad social que los otros dos grupos de edad.

McCann et al. (2004) estudiaron las percepciones sobre la identidad de edad en los jóvenes en distintos países y demostraron que, aunque con variaciones, los jóvenes se sentían muy identificados con su grupo de edad. También Williams, Garret y Tennant (2004) observaron que, en relación a los y a las adolescentes, son más propensos a verse a sí mismos subjetivamente.

Y si la juventud es distinta de la adolescencia, también lo es de la edad adulta (a la que se llega aproximadamente a los 30 años) y que está caracterizada, en las sociedades industriales y post-industriales contemporáneas, por la plena emancipación familiar, independencia económica y creación de la propia vida familiar (Arnett 2007).

Tal y como ha sugerido la literatura en psicología del desarrollo humano (Lightfoot, Cole y Cole 2009), mientras que los adolescentes y los jóvenes se muestran, en general, más propensos a "idealizar" la realidad, los adultos podrían mostrarse más cautos 0 "realistas" en sus juicios sobre ella. En este sentido, Elkind (1967) sugirió que la personalidad del joven guarda ciertas características que le hacen ser más radical en sus enunciados y valoraciones. Más concretamente, postuló la existencia de un egocen- 
trismo de juventud según el cual todo gira alrededor de los propios intereses y, sobre todo, se juzga la realidad por contrastes y oposiciones. Por lo tanto, se podría conjeturar un factor madurativo de desarrollo que hiciera que, con la edad, uno fuera más prudente a la hora de establecer diferencias entre grupos. Es de suponer que entre la juventud y la edad adulta (mediana edad) las valoraciones perceptuales de la realidad sean distintas.

En líneas generales, desde el punto de vista del análisis del contexto que nos aportan la Sociología y la Psicología Social, en las sociedades contemporáneas los y las jóvenes no están casados; viven con los padres y todavía no están asentados laboralmente ni son independientes económicamente. De modo que, por un lado, el proceso emergente de independencia de criterio en relación a las personas adultas por parte de los jóvenes y, por el otro, el impacto de la "modernidad líquida" o la fragilidad en las relaciones humanas y sociales -trabajo, amor, consumo, vivienda, etc.- (Bauman 2007) y la fragmentación identitaria que conllevan las actuales condiciones de incertidumbre sobre el porvenir del proyecto vital -con quién compartiré mi vida, en qué sitio viviré, qué trabajo tendré, etc. (Giddens 1994)-, podrían conducir a experiencias intergeneracionales distintas y, por lo tanto, a modos también distintos de percibir el entorno social y las relaciones entre grupos.

\section{Objetivos}

Nos proponemos analizar la percepción de la vitalidad etnolingüística en una muestra de jóvenes y adultos de Cataluña. Más específicamente, nuestro objetivo es comparar la "vitalidad etnolingüística subjetiva" y los factores asociados, entre jóvenes y adultos, en una muestra total y en un subgrupo "Identidad Catalana" (formada por aquellas personas que tienen el catalán como primera lengua y se identifican como catalanes). Esperamos encontrar diferencias en la percepción intergrupal de la vitalidad etnolingüística entre jóvenes y adultos debido a los cambios suscitados por las nuevas condiciones socioestructurales, así como debido al desarrollo o cambio psicológico que se da en distintos momentos del ciclo vital (Arnett 1007; Bauman 2007; Giddens 1994).

\section{MÉTOdO}

\section{Participantes}

Un total de 527 individuos de entre 20 y 62 años ( $M=40,7 ; S D=11,25)$ participaron en el estudio ( $23 \%$ varones y $77 \%$ mujeres). La submuestra de jóvenes incluía a 268 estudiantes universitarios de entre 20 y 30 años ( $M=27,8 ; S D=3,33$ ), siendo el $21,3 \%$ varones y el $78,7 \%$ restante, mujeres. Mientras que la submuestra de adultos (trabajadores de dos empresas) está formada por 259 personas de entre 35 y 62 años de edad ( $M=44,35$; 
$S D=6,69)$. Los varones constituyeron el $19,6 \%$ de la submuestra y las mujeres el $80,4 \%$. A todos los participantes se les preguntó cuál era su primera lengua (catalán o español) y con que grupo se identificaban (catalán o español) surgiendo una submuestra "identidad catalana" de 301 participantes (152 jóvenes y 149 adultos). Para verificar la fiabilidad de su auto-categorización como catalanes se les suministró un cuestionario de identidad etnolingüística (ID) formado por tres preguntas y extraído de Giles y Johnson (1981): importancia identidad catalana, importancia idioma catalán e importancia de que los hijos aprendan el idioma catalán. El índice alpha de Cronbach de consistencia interna para estos tres ítem fue 0,81 . La ID catalana se mostró alta, próxima a 10 (rango 1-10) en este subgrupo catalanohablante. Al igual que en otros estudios realizados con el mismo instrumento metodológico y en la misma Comunidad Autónoma (Viladot 1993; Viladot y Siguan 1992; Viladot et al. 2007), todos los sujetos de la investigación (jóvenes y adultos) eran, desde el punto de vista social, económico y cultural, de clase media y media-alta.

Instrumento

Para evaluar la VELS se utilizó el "Cuestionario de Vitalidad Etnolingüística" (CVS) desarrollado por Bourhis, Giles y Rosenthal (1981), en su versión validada y adaptada al contexto catalán (Viladot 1993; Viladot y Siguan 1992). El instrumento consta de veinte preguntas dobles y dos preguntas sencillas, sobre el catalán o catalanohablantes y el español o castellanohablantes, con respuestas de continuo semántico con palabras antónimas en los extremos (por ejemplo, ninguna-máxima; nunca-siempre). Los participantes indicaban sus percepciones sobre la vitalidad del catalán y del español en una escala de 10 puntos del 1 al 10, de tal forma que una puntuación alta indica una mayor aprobación del ítem, mientras que una puntuación media (5) representa el equilibrio entre la VELS en las dos lenguas que se estudian. El alpha de Cronbach fue de 0,75 para la totalidad de la escala del catalán y 0,76 para la del español.

Las preguntas del CVS reflejan los factores socio-estructurales de vitalidad propuestos originariamente por Giles, Bourhis y Taylor (1977). Estos son: Factor Estatus (FE) con cinco ítems; Factor Demolingüístico (FD) con seis ítems y Factor Apoyo Institucional (FAI) con ocho ítems (ver tabla 1).

El cuestionario incorpora, además de las descritas en la tabla 1, dos preguntas, también dobles, sobre la fuerza y la actividad presente de la lengua catalana y la lengua castellana, y la fuerza y actividad de estas lenguas dentro de 20 y 30 años (¿Qué fuerza y actividad crees que tienen los catalanohablantes?, ¿y los castellanohablantes? ¿Qué fuerza y actividad crees que tendrán dentro de 20 ó 30 años los catalanohablantes?, ¿y los castellanohablantes?). Preguntas que también utilizaban escalas 1 a 10. El conjunto de estas dos preguntas configura el Factor General de Vitalidad (FGV).

La suma de los factores de vitalidad (FE, FD, FAI y FGV) proporciona la variable "Vitalidad Etnolingüística Subjetiva" (VES). Puesto que Willemyns, Pittam y Gallois (1993), en su versión inglesa, y Viladot (1993), Viladot y Siguan (1992), Viladot, Este- 


\section{Tabla 1.}

\section{Preguntas asociadas a los Factores Demografía, Estatus, Apoyo Institucional.}

\begin{tabular}{|c|c|}
\hline Factor demografía & $\begin{array}{l}\text { - Haz un cálculo de la proporción de la población de Cataluña que componen } \\
\text { los siguientes grupos } \\
\text { - Evalúa el índice de natalidad de cada grupo en Cataluña } \\
\text { - En las partes de Cataluña donde los grupos siguientes se concentran, ¿hasta } \\
\text { qué punto son mayoría o minoría? } \\
\text { - ¿Cuántas personas de cada grupo crees que se instalan (inmigran) en } \\
\text { Cataluña cada año? } \\
\text { - ¿Hasta qué punto crees que los individuos que integran cada grupo se casan } \\
\text { solamente entre ellos? } \\
\text { - De cada grupo, ¿cuántas personas emigran (se van) de Cataluña para vivir } \\
\text { en otros sitios? }\end{array}$ \\
\hline Factor estatus & $\begin{array}{l}\text { - ¿Qué consideración crees que tienen los idiomas siguientes en Cataluña? } \\
\text { - ¿Qué consideración crees que tienen los idiomas siguientes internacional- } \\
\text { mente? } \\
\text { - ¿Hasta qué punto crees que están bien considerados los siguientes grupos } \\
\text { en Cataluña? } \\
\text { - ¿Hasta qué punto crees que los grupos siguientes se sienten orgullosos de su } \\
\text { historia cultural y de lo que han conseguido en Cataluña? } \\
\text { - ¿Hasta qué punto crees que los siguientes grupos son ricos en Cataluña? }\end{array}$ \\
\hline $\begin{array}{l}\text { Factor apoyo } \\
\text { y control } \\
\text { institucional }\end{array}$ & $\begin{array}{l}\text { - ¿Con qué frecuencia crees que se usan los idiomas siguientes en los servi- } \\
\text { cios oficiales de Cataluña (por ejemplo, hospitales, servicios sociales, servicios } \\
\text { municipales)? } \\
\text { - ¿Qué influencia crees que tienen los grupos siguientes sobre los asuntos eco- } \\
\text { nómicos y de negocios en Cataluña? } \\
\text { - ¿Hasta qué punto crees que están bien representados los idiomas siguientes } \\
\text { en los medios de comunicación catalanes (por ejemplo, en la TV, radio, perió- } \\
\text { dicos)? } \\
\text { - ¿Hasta qué punto crees que están bien representados los idiomas siguientes } \\
\text { en el conjunto del sistema educativo en Cataluña (Primaria, Secundaria, Uni- } \\
\text { versidad)? } \\
\text { - ¿Qué poder político crees que tienen estos grupos en Cataluña? } \\
\text { - ¿Hasta qué punto crees que estas lenguas están bien representadas en las } \\
\text { entidades relacionadas con el mundo empresarial? (por ejemplo, en los bancos) } \\
\text { - ¿Con qué frecuencia crees que se utilizan los idiomas siguientes en las iglesias } \\
\text { y sitios de culto en Cataluña? } \\
\text { - ¿Hasta qué punto los grupos siguientes están bien representados en la vida } \\
\text { cultural de Cataluña (por ejemplo, festivales, conciertos, exposiciones de arte)) }\end{array}$ \\
\hline
\end{tabular}

Nota: Las preguntas se responden con una escala de 1 ( $0 \%$, nunca, en disminución, minoría muy pequeña, nada) a 10 (100\%, siempre, en aumento, mayoría muy grande, mucho), en función de cada pregunta, y en relación al español o castellanohablantes y catalán o catalanohablantes. 
ban, Nadal y Giles (2007), en su adaptación al catalán, han validado la estructura del concepto de vitalidad en estos cuatro factores mediante un análisis factorial exploratorio y confirmatorio, hemos obtenido directamente la puntuación media a partir de la suma de los ítems que componen cada factor.

\section{Procedimiento}

La muestra está formada por estudiantes universitarios de distintos cursos de la Facultad de Veterinaria de la Universitat Autónoma de Barcelona (UAB) y por adultos de distintas empresas de Cataluña. Los 527 participantes completaron el CVS en catalán, tardando aproximadamente veinte minutos en rellenarlo. Antes de aplicar el cuestionario se informó sobre las características generales del estudio: "conocer la percepción de vitalidad etnolingüística de una muestra de jóvenes y otra de adultos sobre la lengua catalana y el grupo de catalanohablantes y la lengua castellana y el grupo de castellano hablantes en Cataluña". También se informó sobre el anonimato y la voluntariedad de participar en el estudio. El instrumento fue aplicado entre los meses de febrero y abril de 2008. Una vez obtenidos los cuestionarios rellenados se introdujeron en el programa informático SPSS, versión 15.0, con el objetivo de ser analizados.

\section{Resultados}

Se han llevado a cabo dos grupos de análisis. En primer lugar, se describió la VELS, y los factores asociados, en la muestra total del estudio (los 527 participantes) con el objetivo de comparar los resultados obtenidos entre jóvenes y adultos. Más específicamente, se compararon los grupos en relación al total de la puntuación de la vitalidad subjetiva usando un diseño factorial mixto ANOVA 2x2, utilizando la categoría de edad como factor between subjects (jóvenes versus adultos) y la categoría lengua como factor within subjects (catalán versus español). Este diseño nos permite tener en cuenta la variable edad (jóvenes frente a adultos) y la lengua (catalán frente a español), incorporando dos estrategias de inferencias de hipótesis. Por un lado, una estrategia de comparación entre grupos; por otro lado, una estrategia de comparación intra-sujetos. Dado que en nuestro caso disponemos de dos variables independientes (edad y lengua), podemos utilizar este diseño con el objetivo de estudiar cómo el nivel o valor de una variable (por ejemplo, lengua o edad) genera grupos separados y su efecto se infiere por la comparación entre grupos (en nuestro caso, jóvenes y adultos) o entre sujetos (en nuestro caso, catalán y español).

Se completa el análisis con pruebas post hoc de Tukey y la prueba $t$ de Student. Para saber qué media difiere de qué otra se ha utilizado un tipo particular de contrastes denominado comparaciones múltiples post hoc o comparaciones a posteriori. Estas 
comparaciones permiten controlar la tasa de error al efectuar varios contrastes utilizando las mismas medias, es decir, permiten controlar la probabilidad de cometer errores tipo I (rechazar una hipótesis nula que en realidad no debería rechazarse). En este sentido, dado que se asumen varianzas iguales, se ha utilizado uno de los métodos de mayor aceptación, la prueba post hoc de Tukey. Finalmente, se utiliza la prueba $t$ de Student para muestras independientes con el objetivo de comparar dos medias. Las comparaciones que se hacían eran, por un lado, entre jóvenes y adultos y, por otro, entre castellanohablantes y catalanohablantes.

En segundo lugar, se analizaron las submuestras de jóvenes (152) y adultos (149) que se identificaron como catalanes y tenían el catalán como lengua materna (grupo "Identidad Catalana").

\section{Comparación jóvenes versus adultos en la muestra completa}

Análisis multivariado entre dimensiones de vitalidad subjetiva y variables edad y lengua

Comparando las categorías de edad en relación a las cuatro dimensiones de vitalidad (factor demográfico, factor apoyo institucional, factor estatus y factor general de vitalidad), usando un diseño multivariado $2 \times 2$ (categoría de edad como factor "entre" sujetos y categoría lengua como factor "dentro" sujetos), los resultados rindieron un efecto multivariado significativo para el efecto principal de la categoría de lengua, y el efecto de la interacción entre la edad y la lengua $(F(1.164)=65,47, p<0,001 ; y F=(1.164)=4,98, p<0,03$, respectivamente) pero no para el efecto principal de la categoría de edad, lo que indica que en conjunto la categoría lengua y la interacción entre edad y lengua afectan significativamente a las diferencias encontradas. No obstante, los resultados indican que, aunque ambos grupos percibían la vitalidad del español como inferior a la del catalán, los jóvenes distinguieron de forma más marcada las diferencias en relación a los adultos.

Análisis univariado entre dimensiones de vitalidad subjetiva y variables edad y lengua

En la tabla 2 se muestran las comparaciones entre, lengua (castellanohablantes frente a catalanohablantes) y edad (jóvenes frente a adultos) en relación a la vitalidad subjetiva general total, así como los factores estatus, apoyo y control institucional, demografía, y factor general de vitalidad. Como puede observarse, entre jóvenes y adultos solamente se encuentran diferencias estadísticamente significativas en las medias del factor general de vitalidad. Los adultos perciben más vitalidad de los castellanohablantes en comparación con los jóvenes. Mientras que los jóvenes perciben más vitalidad etnolingüística de los catalanohablantes en comparación con la percepción de los adultos. A pesar de que ambos grupos de edad perciben una vitalidad más elevada de los catalanohablantes en comparación con los castellanohablantes, siendo las diferencias entre grupos lingüísticos más pronunciadas en el caso de los jóvenes. En este sentido, las diferencias entre los castellanohablantes y los catalanohablantes por parte de los jóvenes son esta- 
dísticamente significativas. Esta tendencia se observa en los otros factores. Es decir, en todos ellos (vitalidad etnolingüística subjetiva total, factor estatus, factor apoyo y control institucional, factor demografía) los jóvenes perciben mayor vitalidad de los catalanohablantes en comparación con los castellanohablantes. Ello también sucede con el grupo de adultos, a pesar de que las diferencias son menos significativas.

De modo que el examen de los resultados univariados indica que el efecto principal para la categoría lengua fue significativo para todas las dimensiones. Así, el catalán fue percibido como teniendo una mayor ayuda y control institucional que el español, un mayor estatus social y una mayor demografía. El catalán también fue percibido como teniendo mayor vitalidad general.

Tabla 2.

Vitalidad Subjetiva General, Vitalidad Etnolingüistica Subjetiva Total y Factores estatus, apoyo institucional y demografía de catalanes y españoles en función de la edad (jóvenes y adultos de la muestra total=527).

\begin{tabular}{|c|c|c|c|c|}
\hline & \multicolumn{2}{|c|}{ Jóvenes (n=268) } & \multicolumn{2}{|c|}{ Adultos ( $\mathrm{n}=259)$} \\
\hline & $\begin{array}{l}\text { Castellano } \\
\text { hablantes } \\
M(D E)\end{array}$ & $\begin{array}{l}\text { Catalano } \\
\text { hablantes } \\
M(D E)\end{array}$ & $\begin{array}{l}\text { Castellano } \\
\text { hablantes } \\
M(D E)\end{array}$ & $\begin{array}{l}\text { Catalano } \\
\text { hablantes } \\
M(D E)\end{array}$ \\
\hline Factor General de vitalidad & $5,07+-(1,74)$ & $7,12+-(1,42)$ & $5,81-(1,89)$ & $6,55-(1,61)$ \\
\hline Vitalidad Etnolingüística Subjetiva total & $4,92+(0,95)$ & $6,09+(0,77)$ & $5,18(0,96)$ & $5,83(0,89)$ \\
\hline Factor estatus del grupo y la lengua & $5,27+(1,27)$ & $6,20+(0,91)$ & $5,34+(1,18)$ & $5,92+(1,05)$ \\
\hline Factor apoyo y control institucional & $5,04+(1,26)$ & $6,20+(0,79)$ & $5,26+(1,39)$ & $6,16+(0,92)$ \\
\hline Factor demografía del grupo y la lengua & $4,41+(1,26)$ & $4,91+(0,98)$ & $3,76+(1,09)$ & $5,71+(1,17)$ \\
\hline
\end{tabular}

Nota: La M es la media sobre un rango de 1 a 10; la DE significa Desviación Estándar. Con un (+) se indican diferencias significativas entre catalán y español; con un (-) se indican diferencias significativas entre jóvenes y adultos. Con negrita se indican las medias que se comparan. 
Diferencias entre los items de los factores apoyo y control institucional, estatus y demografía

El examen de los resultados univariados entre grupos (jóvenes versus adultos) indicó diferencias estadísticamente significativas en los ítems índice de natalidad, la estima del grupo en Cataluña, el estatus del grupo como mayoría, y la vitalidad futura. Los tests post hoc de Tukey revelaron que, en lo que respecta al índice de natalidad, los jóvenes percibieron el índice de natalidad catalana como más alto que el índice de natalidad castellana, pero el grupo de adultos no percibió ninguna diferencia en los índices de natalidad de los dos grupos.

Por lo que respecta a la consideración percibida sobre la lengua, los jóvenes creyeron que el castellano gozaba de menor consideración en Cataluña, aunque las diferencias no son estadísticamente significativas. No obstante, hubo efectos principales significativos para el efecto principal de la categoría de lengua en los dos grupos de edad, de tal manera que el catalán fue percibido con una vitalidad más alta por lo que hace referencia al estatus de la lengua y la consideración del grupo catalán en Cataluña, y en el orgullo por los logros culturales; también en la implicación en los servicios de gobierno, temas económicos, educativos, políticos, religiosos, culturales y de riqueza; de índices de natalidad y emigración más altos; así como de vitalidad actual y futura. Cabe destacar que todos los participantes (jóvenes y adultos) sólo clasificaron más alto al castellano que al catalán en lo referente a la inmigración y al estatus del castellano a nivel internacional.

\section{Resumen. Principales resultados de la muestra total}

En definitiva, en comparación con la vitalidad percibida en relación al grupo castellanohablantes, el conjunto de la muestra percibe la vitalidad etnolingüística del grupo catalanohablantes más elevada. Estas diferencias de percepción de la vitalidad en relación a los dos grupos se acentúan significativamente en los jóvenes, especialmente en el factor general de vitalidad ("fuerza y actividad del grupo etnolingüístico" y "fuerza y actividad del grupo etnolingüístico dentro de 20-30 años"). Las diferencias significativas entre los jóvenes y los adultos se encuentran en el total de la vitalidad etnolingüística subjetiva, tanto en los castellanohablantes como en los catalanohablantes y en distintos ítems específicos dentro de los subfactores que componen la escala como: "índice de natalidad de cada grupo en Cataluña", "mayoría/minoría", "consideración de los grupos en Cataluña" y "fuerza y actividad de los grupos en Cataluña dentro de 20-30 años".

Comparación jóvenes versus adultos en el subgrupo "Identidad Catalana" ( $N=301)$

Para examinar cómo la identificación y el uso de la lengua se asociaban a las respuestas de los ítems del CVS, se dividió a los participantes en cuatro subgrupos, tomando como criterios su identificación como catalanes o españoles y si el catalán era su lengua materna o no. Ello nos permitió localizar la submuestra "identidad catalana" formada por 
301 participantes (152 jóvenes y 149 adultos). El análisis del chi-cuadrado indicó que los catalanohablantes tenían más probabilidad de identificarse como catalanes, y los que no hablaban catalán tenían más probabilidad de identificarse como españoles $\left(\chi^{2}(1.150)\right.$ $=61,37, p \cdot<0,01)$.

Análisis univariado entre dimensiones de vitalidad subjetiva y variables edad y lengua

Analizando los resultados hallados en los jóvenes y en los adultos que se identifican como catalanes y tienen el catalán como lengua materna, se observan diferencias estadísticamente significativas en el factor demografía general (en relación a la percepción de la vitalidad etnolingüística de los catalanohablantes). Mientras que los jóvenes puntúan superior en la percepción de los catalanohablantes en relación a los castellanohablantes, la relación se invierte en los adultos que perciben una mayor demografía por parte de los castellanohablantes en relación a los catalanohablantes (ver tabla 3). En los otros factores estudiados (vitalidad etnolingüística subjetiva, factor general de vitalidad, estatus y apoyo y control institucional) se observa una misma tendencia en jóvenes y adultos: consiste en percibir más vitalidad en los catalanohablantes en relación a los castellanohablantes, diferencias que se acentúan en el grupo de los jóvenes, siendo más pronunciadas las diferencias entre catalanohablantes y castellanohablantes. Especialmente significativas en los jóvenes son las diferencias entre grupos lingüísticos en "vitalidad subjetiva general", y en el "factor apoyo y control institucional" (ver tabla 3). Volviendo a la comparación entre jóvenes y adultos, especialmente destacables son las diferencias estadísticamente significativas en "apoyo institucional" (los adultos perciben un mayor apoyo y control institucional de los castellanohablantes en comparación a la percepción de los jóvenes) y "vitalidad etnolingüística subjetiva total" (percibiendo más vitalidad etnolingüística del grupo catalanohablantes por parte de los jóvenes en comparación a la percepción del mismo grupo por parte de los adultos).

Diferencias entre los items de los factores apoyo y control institucional, estatus y demografia

Las diferencias significativas entre grupos de edad (jóvenes versus adultos) se dan en los ítems: "índice de nacimientos" (los adultos perciben menos nacimientos por parte de los catalanohablantes en relación a la percepción de los jóvenes); "mayoría/minoría" (la media de los castellanohablantes según los adultos es significativamente superior a la puntuación media obtenida por los jóvenes); "estima de la lengua en Cataluña" (la percepción de los jóvenes del grupo catalanohablantes es superior en relación a la percepción de los adultos); "estima del grupo en Cataluña" (es superior en el grupo castellanohablantes según la percepción de los adultos en relación a los jóvenes); "educación" (más presencia y apoyo del grupo castellanohablantes en educación según los adultos en relación a los jóvenes); "religión" (más presencia del español en eventos religiosos según la percepción de los adultos); "fuerza y actividad" (más percepción de fuerza y actividad del grupo castellanohablantes por parte de los adultos y más fuerza y actividad de los catalanohablantes por parte de los jóvenes). 
Tabla 3.

Percepción de la vitalidad etnolingüística total y desglosada por factores en función de la edad (jóvenes y adultos de la submuestra "identidad catalana" =301).

\begin{tabular}{|c|c|c|c|c|}
\hline & \multicolumn{2}{|c|}{ Jóvenes (n=152) } & \multicolumn{2}{|c|}{ Adultos $(n=149)$} \\
\hline & $\begin{array}{l}\text { Castellano } \\
\text { hablantes } \\
\mathrm{M}(\mathrm{DE})\end{array}$ & $\begin{array}{l}\text { Catalano } \\
\text { hablantes } \\
\mathrm{M}(\mathrm{DE})\end{array}$ & $\begin{array}{l}\text { Castellano } \\
\text { hablantes } \\
\mathrm{M}(\mathrm{DE})\end{array}$ & $\begin{array}{l}\text { Catalano } \\
\text { hablantes } \\
\mathrm{M}(\mathrm{DE})\end{array}$ \\
\hline Factor de vitalidad general & $5,16+(1,82)$ & $6,95+(1,51)$ & $6,00(1,89)$ & $6,32(1,68)$ \\
\hline $\begin{array}{l}\text { Vitalidad Etnolingüística } \\
\text { Subjetiva total }\end{array}$ & $4,86(0,96)$ & $6,15-(0,80)$ & $5,32(0,96)$ & $5,69-(0,93)$ \\
\hline Factor estatus del grupo y la lengua & $5,11(1,36)$ & $6,28(0,89)$ & $5,23(1,29)$ & $5,86(1,13)$ \\
\hline Factor apoyo y control institucional & $4,89+-(1,35)$ & $6,24+(0,86)$ & $5,71-(1,29)$ & $5,98(0,93)$ \\
\hline Factor demografía del grupo y la lengua & $4,63(1,23)$ & $5,19-(0,97)$ & $4,75(1,18)$ & $4,54-(1,16)$ \\
\hline
\end{tabular}

Nota: La M es la media sobre un rango de 1 a 10; la DE significa Desviación Estándar. Con un (+) se indica diferencias significativas entre catalán y español; con un (-) se indica diferencias significativas entre jóvenes y adultos. Con negrita se indican las medias que se comparan.

Resumen. Principales resultados submuestra "identidad catalana"

En general y, al igual que en la muestra total, se observan:

a) medias más altas (estadísticamente significativas) de la vitalidad etnolingüística de los catalanohablantes percibida por los jóvenes, en comparación con los adultos, y

b) medias más altas (estadísticamente significativas) de la vitalidad etnolingüística de los castellanohablantes percibida por los adultos, en comparación con los jóvenes.

\section{Discusión}

Los resultados del siguiente estudio permiten sugerir patrones de percepción de vitalidad etnolingüística "similares" y "distintos" entre jóvenes y adultos. "Similares" ya que en líneas generales en ambos grupos de edad se percibe que los catalanohablantes gozan de más vitalidad etnolingüística en Cataluña en comparación con los castellanohablan- 
tes. Ello se sitúa en la línea de estudios previos (Atkinson 2000; Romay, García-Mira y Azurmendi 1999; Giles y Viladot 1994; Viladot 1993; Viladot y Siguan 1992; Viladot et al. 2007; Ytsma, Viladot y Giles 1994) que indican una mayor percepción de vitalidad etnolingüística del grupo catalanohablantes en relación a los castellanohablantes en Cataluña. "Distintos", y aquí se encuentra la novedad del estudio realizado, ya que en el grupo de jóvenes las diferencias entre la vitalidad etnolingüística percibida de los catalanohablantes y castellanohablantes son más pronunciadas que en el grupo de adultos. Es decir, los jóvenes perciben una mayor diferencia entre el grupo castellanohablantes y catalanohablantes, especialmente en el factor de vitalidad subjetiva general ("fuerza y actividad del grupo" y "fuerza y actividad del grupo dentro de 20-30 años") a favor del grupo catalanohablantes. En los adultos participantes la discriminación entre los dos grupos etnolingüísticos es ligera y no significativa, en líneas generales. Podemos conjeturar que dichas diferencias intergeneracionales se podrían explicar, en parte, y sólo en parte, por factores psicológicos.

No obstante, estas explicaciones corren el riesgo de obviar la dimensión social y caer en estereotipos y generalizaciones del tipo: los jóvenes son más radicales en sus percepciones intergrupales y los adultos son más críticos, cautos y maduros. Ciertamente, a menudo constatamos que algunas investigaciones sobre el colectivo juvenil ofrecen una imagen distorsionada y con tendencia a identificar a los jóvenes con valores, hechos y comportamientos negativos. Estos estudios apoyan el proceso de construcción de la imagen de una juventud extraña y estrambótica ajena a los valores y procesos sociales imperantes. Ello no significa que, atendiendo a los estudios de la Psicología Evolutiva, no consideremos que la distorsión perceptual de los jóvenes (relativa a los adultos) entre la vitalidad de los catalanohablantes y los castellanohablantes encontrada en el presente trabajo no sea en parte debido, a la variable evolutiva de la edad. Sin embargo, para explicar la comunicación intergrupal la naturaleza del contexto tiene una gran importancia. Teniendo en cuenta que estamos analizando las percepciones sobre vitalidad cultural y lingüística de los dos grupos etnolingüísticos en Cataluña históricamente en contacto, ¿cómo afectará el contexto actual que ya hemos descrito en el grupo minoritario catalanohablante? ¿Hasta qué punto factores identitarios son responsables del patrón encontrado, descrito anteriormente?

En relación con la submuestra de jóvenes que se identifican como catalanes y tienen el catalán como lengua materna se sigue el mismo patrón anterior, pero aquí los jóvenes (comparativamente a las percepciones de los adultos) perciben la vitalidad de los catalanohablantes mucho más elevada que la de los castellanohablantes.

Es posible analizar, sin embargo, desde otra vertiente conceptual las diferencias halladas en la percepción de los jóvenes y los adultos. La literatura sobre comunicación intergrupal ha mostrado cómo los factores cognitivos y motivacionales pueden ayudar a explicar la acentuación o la atenuación de las vitalidades diferenciales del endogrupo y el exogrupo en situaciones multilingües (por ejemplo: Sachdev y Bourhis 1993; Harwood, Giles y Bourhis 1994). Muy probablemente los jóvenes de nuestro estudio han exagerado los méritos de la vitalidad de su grupo etnolinguístico atenuando la vita- 
lidad del exogrupo castellanohablante, y así mantener una distintividad positivamente valorada. Ello se puede explicar a la luz de la teoría de la identidad social (Tajfel 1981; Tajfel y Turner 1986). Esta teoría postula que las personas estamos motivadas para mantener 0 aumentar un sentido positivo de nuestra identidad social de tal modo que en las interacciones con otros grupos realizamos comparaciones en relación con el estatus relativo de nuestro grupo. Fruto de estas comparaciones con el exogrupo puede surgir la percepción de una identidad social satisfactoria, en cuyo caso las estrategias se orientarán a conservar la propia superioridad o a extenderla. Pero también la percepción de la identidad social puede ser insatisfactoria, en cuyo caso se desencadenará el uso de estrategias para conseguir cambios que restablezcan la percepción positiva de estatus.

Si un grupo de fuerte identidad es amenazado por un grupo ajeno es probable que los miembros de ambos grupos perciban los encuentros en términos intergrupales y utilizen estrategias de gestión de su identidad. Es decir, los grupos etnolingüísticos buscarán establecer una distintividad etnolingüística positiva a través del establecimiento de comparaciones sociales favorables con los exogrupos relevantes. Una comunicación intergrupal no convergente 0 divergente puede ser especialmente poderosa para los grupos etnolingüísticos y nacionales que intentan mantener su identidad social, usando mecanismos identitarios y lingüísticos como táctica simbólica para manterner su especificidad como grupo, a la vez que rechazan normas ajenas a su endogrupo (Fishman 2001; Giles y Coupland 1991). A nivel intergrupal, la motivación para alcanzar o mantener una identidad social positiva, como ha propuesto la teoría de la identidad social, ha sido más evidente en estudios que han demostrado la divergencia lingüística y el mantenimiento de la lengua en distintas partes de mundo. Diversos estudios han verificado que la identificación etnolingüística positiva del grupo está relacionada con la evaluación positiva de su vitalidad etnolingüística.

Quizás la crisis económica actual, sumada al mundo global del siglo XXI, ha comportado para los jóvenes un mayor grado de incertidumbre sobre el futuro. Harwood, Giles y Bourhis (1994) apuntan que los niveles de incertidumbre social pueden ser un importante determinante de los perfiles de vitalidad percibida. Argumentan con apoyo empírico que la incertidumbre social -ver Viladot (2008) para una revisión de la teoría de la gestión de la ansiedad y la incertidumbre de Gudykunts- y las percepciones de inestabilidad tienden a hacer más sobresalientes las identidades sociales de los individuos. En este sentido, estudios anteriores a la actual crisis económica muestran diferencias perceptuales endogrupo/exogrupo en Cataluña no tan pronunciadas como las encontradas en este estudio (ver Viladot 1993; Viladot y Siguan 1992). A pesar de que los enormes cambios sociales y económicos que comporta la "modernidad líquida" de Bauman (2007) tienen diferentes implicaciones para los distintos grupos de la sociedad, estos cambios pueden llevar a la necesidad de un incremento intragrupal focalizado en la valoración de la vitalidad. Es decir, para mantener una identidad social positivamente valorada los miembros del grupo se disociaran de otros grupos en un grado mayor que en tiempos más estables. De este modo revalorizan la vitalidad amenazada y, en consecuencia, la autoestima o la identidad social del propio grupo (para estrategias de gestión 
de la identidad social y etnolingüística, ver, por ejemplo, Viladot 2008). En este sentido, los jóvenes que se identifican como catalanes podrían sentir amenazada su identidad etnolingüística. Ello puede activar procesos de discriminación exogrupal con el objetivo de revalorizar la autoestima e identidad etnolingüística del grupo. Además, la incertidumbre en relación al futuro que depara el escenario sociopolítico actual y que caracteriza a los jóvenes de hoy día (Arnett 2007) podrían motivar la exageración o radicalización de la percepción de la vitalidad etnolingüística de los grupos catalanohablantes y castellanohablantes.

No existen estudios previos al respecto de modo que no nos ha sido posible comparar los resultados obtenidos con otros trabajos. Naturalmente, se requiere trabajo futuro en contextos similares que proporcionen una amplia comprensión de los resultados obtenidos en la cohorte de los jóvenes. Sin embargo, cabe insistir en que la incertidumbre social es vista como un factor principal que tiene una influencia decisiva en el grado de consenso del grupo sobre las percepciones de vitalidad en las relaciones intergrupales.

Futuras investigaciones deberían aclarar las diferencias halladas en este estudio en relación a la percepción de los jóvenes y los adultos utilizando muestras más amplias, así como utilizar otras fuentes de información derivadas, por ejemplo, de la metodología cualitativa. Además, recientemente, Abrams, Barker y Giles (2009) han propuesto ciertas modificaciones del instrumento utilizado (el Cuestionario de Vitalidad Etnolingüística Subjetiva) que cabría adaptarse, en su versión adaptada al contexto catalán, e implementarse en otras muestras con el objetivo de validar de nuevo el instrumento.

En definitiva, pensamos que el estudio aporta una nueva línea de investigación en el campo de estudio de la vitalidad etnolingüística consistente en la comparación intergeneracional de grupos de edad. Ello debe permitir obtener una descripción de la percepción de vitalidad más ajustada a la realidad, teniendo en cuenta muestras más amplias que las habitualmente utilizadas (estudiantes universitarios).

\section{RefERENCIAS BibliográfICAS}

Abrams, J. R., V. Barker y H. Giles. 2009. "An Examination of the Validaty of the Subjective Vitality Questionnaire." Journal of Multilingual \& Multicultural Development 30:59-72.

Allard, R. y R. Landry. 1994. "Subjective Ethnolinguistic Vitality: A Comparison of Two Measures." Internacional Journal of the Sociology of Language 108:117-144.

Atkinson, D. 2000. "Minoritisation, Identity and Ethnolinguistic Vitality in Catalonia." Journal of Multilingual and Multicultural Development 21:185-197.

Arnett, J. J. 2000. "Emerging Adulthood: A Theory of Development from the Late Teens Through the Twenties." American Psychologist 55:469-480.

Arnett, J.J. 2007. Adolescence and Emerging Adulthood: A Cultural Approach. Washington, DC: Prentice-Hall INC. 
Azurmendi, M. J. y R. Bourhis. 1998. "Presentación: Proyecto de Investigación ICYCLABE-1996: Identidades culturales y lingüísticas en las Comunidades Autónomas bilingües (CAB) de España." Revista de Psicología Social 13:545-558.

Baldaquí, J. M. 2004. La percepció de la vitalitat etnolingüística dels joves de l'Alicantí. Alacant y Barcelona: Publicacions de l'Abadia de Montserrat.

Bauman, Z. 2007. Temps líquids: Viure en una epoca d'incertesa. Barcelona: Viena.

Bourhis, R. Y. y A. Maass. 2005. "Linguistic prejudice and stereotypes." Pp. 1587-1602 en Sociolinguistics. An International Handbook of the Science of Language and Society. editado por . Ammon, N. Dittmar, K, J. Mattheier y P. Trudgill. Berlin y New York: Walter De Gruyter.

Bourhis, R., H. Giles y D. Rosenthal. 1981. "Notes on the Construction of a Subjective Vitality Questionnaire for Ethnolinguistics Groups." Journal of Multilingual and Multicultural Development 2:144-155.

Cenoz, J. y J. F. Valencia. 1993. "Ethnolinguistic Vitality, Social Networks and Motivation in Second language acquisition: Some data from the Basque country." Language, Culture, and Curriculum 6:113-127.

Clément, R. y R. Y. Bourhis. 1996. "Bilingualism and Intergroup Communication." International Journal of Psycholinguistics 12:171-191.

Elkind, D. 1967. "Egocentrism in adolescence." Child Development 38:1025-1034.

Fishman, J. 2001. Handbook of Language and Ethnic Identity. Nueva York: Oxford University Press.

Giddens, A. 1997. Modernidad e identidad del yo: el yo y la sociedad en la época contemporánea. Barcelona: Península.

Giles, H. y N. Coupland. 1991. Language: Contexts and Consequences. Keynes: Open University Press.

Giles, H. y P. Johnson. 1987. "Ethnolinguistic identity theory: A social psychological approach to language maintenance." International Journal of the Sociology of Language 68:69-99.

Giles, H y M. A. Viladot. 1994. "Ethnolinguistic Differentiation in Catalonia." Multilingua 13:301-312.

Giles, H., R. Bourhis y D. M. Taylor. 1977. "Towards a Theory of Language Interethnic Group Relations." Pp. 307-348 en Language, Ethnicity and Intergroup Relations, editado por H. Giles. Londres: Academia Press.

Giles, H., D. Rosenthal y L. Young. 1985. "Perceived ethnolinguistic vitality: The Anglo and GreekAustralian setting." Journal of Multilingual and Multicultural Development 6:253-269.

Harwood, J., H. Giles y R. Bourhis. 1994. "The genesis of vitality theory: Historical patterns and discoursal dimensions." International Journal of the Sociology of Language 108:167-206.

Harwood, J., H. Giles, R. Clément, H. Pierson y S. Fox. 1994. "Perceived Vitality of Age Categories in California and Hong Kong." Journal of Multilingual and Multicultural Development 15:37-46. 
Karahan, F. 2004. "Ethnolinguistic vitality, attitudes, social network and code-switching: The case of Bosnian-Turks living in Sakarya." International Journal of Sociology of Language 165:59-92.

Komondouros, M. y L. Mcentee-Atalianis. 2007. "Language attitudes, shift and ethnolinguistic vitality of the Greek Orthodox community in Istanbul." Journal of Multilingual and Multicultural Development 28:365-384.

Kraemer, R. 1992. "Ethnolinguistic vitality perceptions in Israel in the wake of the Intifada." Journal of Multilingual and Multicultural Development 13:482-503.

Kraemer, R., E. Olshtain y S. Badier. 1994. "Ethnolinguistic Vitality, Attitudes, and Networks of Linguistic Contact: The case of the Israeli Arab minority." International Journal of Sociology of Language 108:79-95.

Landry, R. y R. Allard. 1994. "Disglossia, Ethnolinguistic Vitality, and Language Behavior." International Journal of the Sociology of Language 108: 15-42.

Lighthoot, C., M. Cole y S. Cole. 2009. The Development of Children. Nueva York: Worth Publishers.

McCann, C. C. 2000. "Reviewing Ethnolinguistic Vitality: The Case of Anglo-Nigerian Pidgin." Journal of Sociolinguistics 4:458-47.

McCann, R. et al. 2004. "Cultural and Gender Influences on Age Identification." Communication Studies 55:88-105.

McConnell, G. 1991. A Macro-sociolinguistic Analysis of Language Vitality: Geolinguistic Profiles and Scenarios of Languages Contact in India. Sainte-Foy: Les presse de I'Université Laval.

Pierson, H. D. 1994. "Ethnolinguistic Vitality during a Period of Decolonization without Independence: Perceived vitality in Hong Konk." Internacional Journal of the Sociology of Language 108:43-62.

Romay, J., R. García-Mira y M. J. Azurmendi. 1999. "Identidad etnolingüística y vitalidad etnolingüística en las Comunidades Autónomas Bilingües (CAB) de España." Revista de Psicología Social 14:87-106.

Ros, M., C. Huici e I. Cano. 1994. "Ethnolinguistic Vitality and social identity: their impact on ingroup bias and social attribution." International of Sociology of Language 3:145-166.

Ros, M. M. J. Azurmendi, R. Y. Bourhis e I. García. 1999. "Identidades etnolingüísticas en las Comunidades Autónomas Bilingües (CAB) de España: antecedentes y consecuencias." Revista de Psicología Social 14:69-86.

Sachdev, I. y R. Y. Bourhis. 1993. "Ethnolinguistic vitality and social identity." Pp. 33-51 en Group motivation: Social Psychological Perspectives, editado por M. A. Hogg y D. Abrams. Hemel Hempstead: Harvester Wheatsheaf.

Tajfel, H. 1981. Human Groups and Social Categories: Studies in the Social Psychology. Cambridge: Cambridge University Press.

Tajfel, H. y J. C. Turner. 1986. "The Social Identity Theory of Intergroup Behavior." Pp. 7-24 en The Social Psychology of Intergroup Relations, editado por H. Tajfel. Chicago: Nelson-Hall. 
Triandis, H. C. 1994. Culture and Social Behavior. Nueva York: McGraw-Hill.

Viladot, M. A. 1993. "Vitalidad etnolingüística subjetiva y uso de la lengua en el contexto catalán." Revista de Psicología Social Aplicada 3:27-46.

Viladot, M. A. 1994. "Percepción de vitalidad de las cohortes de edad en Cataluña". (Documento de trabajo no publicado)

Viladot, M. A. 2008. Lengua y comunicación intergrupal. Barcelona: UOC.

Viladot, M. A. y M. Siguan. 1992. "Aproximación empírica a la teoría de la identidad etnolingüística en el contexto catalán." Anuario de Psicología 52:79-93.

Viladot, M. A., M. Esteban, J. M. Nadal y H. Giles. 2007. "Un estudio sobre identidad y percepción de vitalidad etnolongüística en Catalunya." Revista de Psicología Social Aplicada 17:223-247.

Willemyns, M., J. Pittam y C. Gallois. 1993. Perceived Ethnolinguistic Vitality of Vietnamese and English in Brisbane: A Confirmatory Factor Analysis. Australia: University of Queensland.

Williams, A., P. Garret y R. Tennant. 2004. "Young adults' perceptions of communication with peers and adolescents'." Pp. 35-52 en Language and Social Psychology, editado por C. Candling y C. Chiu. Hong Kong: City University of Hong Kong Press.

Young, L., H. Giles y H. Pierson. 1986. "Sociopolitical change and perceived vitality." International Journal of Intercultural Relations. 10:459-469.

Ytsma, J., M. A. Viladot y H. Giles. 1994. "Ethnolinguistic vitality and ethnic identity: Some Catalan and Frisian data." International Journal of the Sociology of Language 108:63-78.

MARIA ÀNGELS VILADOT PRESAS es Licenciada en Filosofía y Letras (sección Psicología) por la Universitat Autònoma de Barcelona, de la cual fue profesora adjunta. Doctora en psicología y ciencias de la educación por la Universitat de Barcelona. Actualmente es profesora adscrita a los estudios de ciencias de la información y comunicación y a los estudios de psicología y ciencias de la educación en la Universitat Oberta de Catalunya, miembro de la International Communication Association (ICA) y vocal del Consejo Social de la Lengua Catalana (Generalitat de Catalunya). Dirige la colección "Nuevo punto de vista" de la Editorial Aresta y forma parte del consejo editorial de la colección Comunicación y Sociedad (Editorial UOC y Editorial Aresta) dirigida por Howard Giles de la University of California, Santa Barbara. Colabora asiduamente con el departamento de comunicación de la University of California, Santa Barbara. Su ámbito de investigación gira alrededor de la comunicación intergrupal e intercultural. 
MOISĖS ESTEBAN GUITART es licenciado en Filosofía y en Psicología por la Universitat de Girona. Doctor en psicología (programa de Doctorado Interuniversitario en Psicología de la Educación coordinado por la Universitat de Barcelona). Ha ampliado estudios en la Universidad de Salamanca, la Universidad Intercultural de Chiapas, la Leeds University, el Institute for Cultural Research and Education y ha sido profesor visitante en el College of Education de la University of Arizona. Actualmente es profesor lector en la Universitat de Girona. Es miembro de la International Society for Cultural and Activity Research (ISCAR). Su principal línea de investigación son los procesos de construcción identitaria en contextos multiculturales.

\section{RECIBIDO: $10 / 05 / 2009$}

ACEPTADO: $17 / 06 / 2010$

Publicado on-line: 25/11/2010 
